\title{
Rapidly Progressive Glomerulonephritis
}

National Cancer Institute

\section{Source}

National Cancer Institute. Rapidly Progressive Glomerulonephritis. NCI Thesaurus. Code C35264.

Inflammation of the glomeruli that is characterized by a rapid loss in renal function with glomerular crescent formation observed on biopsy; it is often seen in patients with concomitant autoimmune disease, like Goodpasture's syndrome or systemic lupus erythematosus. 